\title{
The potential role of indoleamine 2,3 dioxygenase (IDO) as a predictive and therapeutic target for diabetes treatment: a mythical truth
}

\author{
Babak Baban • W. Todd Penberthy • \\ Mahmood S. Mozaffari
}

Received: 14 January 2010 / Accepted: 4 February 2010 /Published online: 19 March 2010

(C) European Association for Predictive, Preventive and Personalised Medicine 2010

\begin{abstract}
Type 1 diabetes (T1D) is an autoimmune disease in which a T-cell-mediated reaction demolishes insulinproducing cells of pancreatic islets. Inadequacy of insulin therapy has motivated research focused on mechanisms by which autoimmune reactions can be suppressed. In recent years, the role of indoleamine 2,3 dioxygenase (IDO) in regulation of immune system has been extensively investigated. Initially, IDO was recognized as a host defense mechanism. However, recent studies have suggested an immunomodulatory role for IDO which may contribute to the induction of immune tolerance. In this review, we concentrate on the role of IDO in several pathologic conditions with a focus on T1D to rationalize our hypothesis regarding the potential for inclusion of IDO in certain therapeutic strategies aimed at early detection, treatment or ideally cure of chronic and autoimmune diseases such as T1D.
\end{abstract}

Keywords Individual tolerance $\cdot$ Predictive diagnostic . Targeted treatment $\cdot$ IDO $\cdot$ Type 1 diabetes

\section{Introduction}

Diabetes is a group of heterogeneous diseases marked by high levels of blood glucose, resulting from defects in insulin

B. Baban $(\bowtie) \cdot$ M. S. Mozaffari

Department of Oral Biology, School of Dentistry,

Medical College of Georgia Augusta,

Georgia 30912, USA

e-mail: bbaban@mcg.edu

W. T. Penberthy

University of Cincinnati,

Building C Room 217A, 2180 E. Galbraith Road Cincinnati,

$\mathrm{OH} 45237$, USA production, insulin action, or both. Diabetes can lead to serious complications and premature death, but diabetic patients can take steps to control the disease and lower the risk of complications. According to the World Health Organization, over 180 million people worldwide suffer from diabetes. Its incidence is increasing rapidly and it is estimated that by the year 2030, this number will almost double.

Type 1 diabetes (T1D), previously called insulin-dependent diabetes mellitus (IDDM) or juvenile-onset diabetes, accounts for $10 \%$ of all diagnosed cases of diabetes. It develops when the body's immune system destroys pancreatic beta cells, thereby causing hypoinsulinemia. To survive, people with T1D must receive exogenous insulin, delivered by injection or a pump. This form of diabetes usually strikes children and young adults, although disease onset can occur at any age. Risk factors for T1D may be autoimmune, genetic, or environmental.

Despite advances in our understanding and treatment of T1D, there is no known way to cure or prevent T1D and its severe complications. However, it is obvious that because of its autoimmune nature, any protocol for treatment of T1D requires to be associated with a meticulous manipulation of immune response and interactions.

In recent years, the activity of indoleamine 2,3-dioxygenase (IDO), a tryptophan-degrading enzyme has attracted significant attention. This is due to a unique dichotomy in IDO's function. IDO was originally recognized for its direct role in the immune responses against pathogens and infectious agents. However, increasing evidence imply that IDO may have an immunoregulatory capacity enabling IDO to contribute in creating immune tolerance and inhibition of effective immune responses.

Our focus in this review is on recent advances and findings about immunomodulatory features of IDO and its interaction with the immune system. Summarizing these 
data then allows us to suggest a hypothetical novel role for IDO as a predictive marker which may help in devising better therapeutic strategies to treat and control chronic and autoimmune diseases such as T1D.

\section{Indoleamine 2,3-dioxygenase (IDO)}

Indoleamine 2,3-dioxygenase (IDO) is a cytosolic hemecontaining enzyme that catalyzes the first, and rate-limiting step of oxidative tryptophan catabolism (Figs. 1 and 2) [1].

IDO is inducible in many tissues and cell types, but most prominently, in antigen presenting cells (APC). Two closely linked, homologous genes (IDO1, IDO2) located in syntenic regions of chromosome 8 in humans and mice encode IDO proteins [2-4]. All mammalian IDO genes studied to date possess one, or more interferon (IFN) response elements (ISRE, GAS) in their promoter regions, and IFNs produced at local sites of inflammation are potent inducers of IDO in several cell types such as some dendritic cells (DCs), macrophages, epithelial, trophoblasts and endothelial cells [5-8]. IDO enzyme activity catabolizes the essential amino acid tryptophan (Fig 2) [9], consumes superoxide radicals, and produces tryptophan metabolites known as kynurenines.

IDO activity is measured by assessing kynurenine levels in serum, or in tissues by HPLC analysis. Functional IDO activity depends on heme and substrate supply, redox potentials, and the absence of heme-toxins such as nitric oxide, as well as other poorly defined post-translational modifications [10, 11]. Hence, it is important to measure enzyme activity, or IDO-mediated effects on T cell responses, as IDO gene and protein expression though necessary, may not be sufficient to drive IDO enzyme activity.

\section{Immunological significance of IDO}

Pfefferkorn and colleagues initially demonstrated that an IDO dependent mechanism is responsible for growth

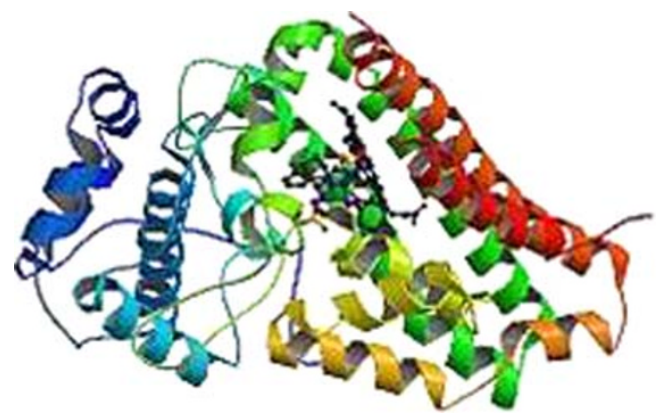

Fig. 1 Ribbon representation of the overall structure of human IDO (Protein Data Bank) inhibition of intracellular parasite, Toxoplasma gondii. This finding clearly categorized IDO as a host defense mechanism of innate responses [12].

The enzymatic activity of IDO and its non-immunological role have been clearly described, however, understanding of mechanisms by which IDO exerts its impacts on immune system still remain an elusive scientific goal.

It was 1998 when the discovery by Munn and his colleagues transformed the existing knowledge about IDO [13]. Their studies showed that IDO plays an essential role in protecting embryo from rejection in a murine allogeneic pregnancy model. Importantly, inhibition of IDO activity by its pharmacologic inhibitor, 1 methyl tryptophan (1MT), resulted in $\mathrm{T}$ cell mediated rejection of fetuses. These findings not only proposed an immunological role for IDO, but also suggested a possible novel role for IDO in generating the immune tolerance and regulating the immune responses. Furthermore, these findings were in contradiction with IDO's originally and widely accepted function as a host defense mechanism. Therefore, IDO seems to act in a dual manner: a) it exerts antimicrobial effects by inhibiting the growth of microorganisms and stimulating the immune responses, and b) several studies suggest that IDO may be involved in activation of regulatory immune responses and creation of immune tolerance.

In recent years, several theories have been suggested to characterize the role of IDO in normal and disease conditions. Müller et al. propounded a general proposal for IDO's interactions with the immune system [14]. In their model, both assumed immunological roles of IDO, stimulatory and regulatory, are concurrent and linked to each other. In other words, they suggest that the immune system, in an IDO mediated pathway, firstly reacts to the infectious agent in order to inhibit the growth and progression of the pathogen and disease. Later, in the second phase, the IDO-mediated regulatory immune mechanism gets activated to protect the host from any possible over reaction induced by the effector wing of the immune system. Although, the exact mechanisms for this proposal needs to be investigated much further, however, different factors such as host microenvironment, physical and chemical conditions are involved in triggering this conceptual process. Other hypotheses to explain the mechanisms by which IDO may apply its supposedly regulatory impact on the immune system can be consolidated into two main groups. The first group proposes that tryptophan depletion is the key mechanism by which IDO exerts its possible regulatory function on the immune system while the second group suggests that accumulation of tryptophan metabolites in the microenvironment is responsible for IDO's immunoregulatory feature.

Further detailed in vivo and in vitro studies are required before any of these models, described above, can be 
Fig. 2 A schematic diagram of the kynurenine pathway [9]

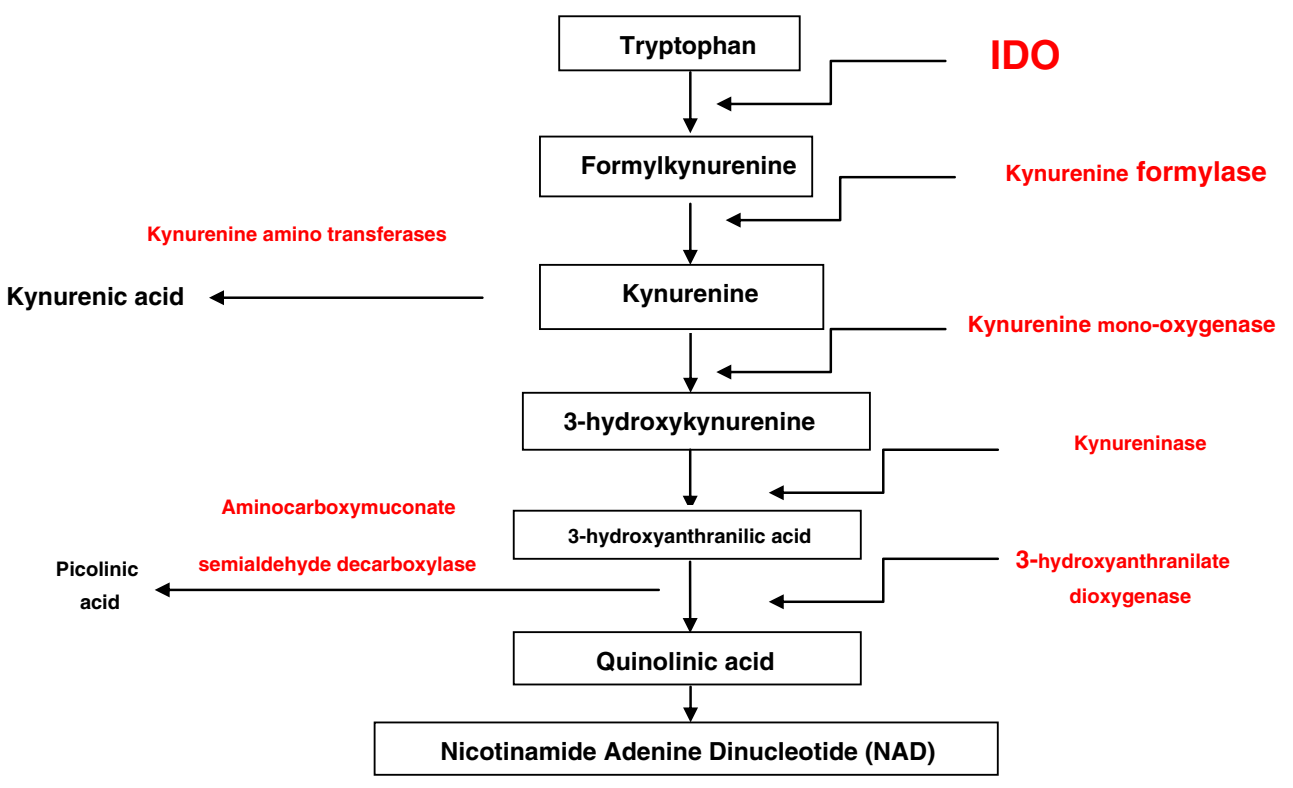

referred to as the main pathway for the presumed IDOmediated regulation of the immune system. However, taken together, these theories along with increasing supporting data are indicators of the possible significant impact of IDO on the immune system. Furthermore, these hypotheses are offering a rationale for more extended investigation on possible IDO's role as an immunomodulatory agent with potential applications in experimental and clinical studies.

\section{Pathological and prognostic significance of IDO}

IDO and its correlation with initiation, progression and clinical manifestation of diseases, have recently become the focus of several studies. In fact, increasing evidence suggests a critical immunoregulatory function for IDO and supports the notion that IDO may have the capacity to affect the course of a variety of pathological conditions such as autoimmunity, infections, cancer and transplantation.

\section{IDO and infection}

Preliminary evidence to show the effects of IDO on infections was that growth of certain intracellular pathogens such as Toxoplasma gondii was inhibited in an IDO dependent manner. A recent study by Spekker and colleagues suggested that IDO is responsible for the suppression of Neospora caninum growth [15]. Other studies on Toxoplasma gondii suggested that its growth could be contained when certain immune cells including dendritic cells were actively expressing IDO. Further studies have proposed that enhanced IDO expression resulted in resolving of bacterial infections. Same results were obtained in models when infections were caused by Mycobacterium tuberculosis and Bartonella henselae $[16,17]$. Mackler and his colleagues demonstrated that higher expression of IDO was associated with remission of bacterial infection, Listeria monocytogenes, in the murine placenta [16, 18-21]. Their findings suggested that IDO contributed to the lessening of the bacterial infection progression while at the same time maintaining a barrier to $\mathrm{T}$ cells whose presence might result in fetal rejection. These results clearly indicated a paradox in IDO function which propose that IDO acts in a way to regulate a fine balance between inflammatory responses, required for protection and immune privilege which is pivotal to contain the excessive inflammatory responses. Furthermore, other recent studies have reported similar findings of higher expression level along with a dichotomy in function for IDO in fungal infection models [22, 23].

\section{IDO and cancer}

The possibility of a pathologic relation between IDO and cancer was initially proposed by Uyttenhove et al. when they showed that IDO could lessen the $T$ cell responses against tumor in a murine model [24]. Furthermore, Munn et al. were able to detect IDO in tumor draining lymph nodes where tumor antigens first drain and tumor-derived DCs first migrate; IDO was expressed by both tumor cells and dendritic cells [25]. Some reports have shown that tumor cells express IDO, and moreover, transfection of IDO into tumor cells was able to block anti-tumor responses by immune cells. The exact mechanisms by which IDO can prevent tumor rejection is not fully characterized, however, several studies have hypothesized different theories attempting to characterize the mechanism(s) by which 
IDO may protect tumor from rejection by effector immune responses. One of the recent proposals is considering a genetic pathway to explain the interaction between cancer and assumed IDO immunosuppressive feature. Based on this theory, IDO is contained by cancer suppression gene Bin1, which is attenuated in many human malignancies. Data from this particular study showed that loss of Bin1 in knockout mouse resulted in elevation of IDO expression, driving escape of oncogenically transformed cells from $\mathrm{T}$ cell-dependent antitumor immunity which could be reversed by using IDO inhibitor [26]. Therefore, these data suggest that deficiency in Bin1 boosts cancer's ability of ignoring the immune system by deregulating IDO and that IDO inhibitors may improve responses to cancer chemotherapy. However, data from another study demonstrated no relation between IDO and Bin1 [27]. Moreover, the same study showed that neither IDO nor Bin1 had any correlation with survival rate. Therefore, in a very paradoxical pattern compared with observations from previous studies, these new data suggested that it is very unlikely to establish any relation between IDO activity and progression of certain types of cancer. These disagreements may relate to differences in experimental design and research protocols. More investigations are required before any mechanism(s) for IDO and its impact on immune system could be determined.

Several studies have suggested that IDO is expressed and dictates its supposedly tolerogenic effect on immune system during cancer development at two sites [16]. The first is at the tumor site where hypothetically tryptophan depletion and induction of anti-proliferatory metabolites suppress $\mathrm{T}$ cell responses against tumor, creates a microenvironment in which tumor progression and development of cancer occurs. The second site of IDO expression is at the level of tumor draining lymph nodes where a specific subset of dendritic cells, plasmacytoid dendritic cells (PDCs), are expressing IDO; it is suggested that at this level, IDO exerts its immunosuppressive impacts on T cells which results in tumor growth. Interestingly, it was shown that higher number of IDO expressing dendritic cells in draining lymph nodes of melanoma patients is directly correlated with adverse development of the disease [25]. Similar results were observed by Lee et al. in an independent study [28]. In fact, results from several cancer studies suggest a direct correlation between the level of IDO activity and tumor progression and poor prognosis in patients with certain types of cancer. Urakawa and colleagues [29] have demonstrated that higher level of IDO expression is directly correlated with metastasis and a poorer clinical outcome and survival rate in osteosarcoma. In another report, Brandacher et al. [30] have suggested that IDO significantly contributes to disease progression, significant reduction in intratumoral effector $\mathrm{T}$ cells and overall survival in patients with colorectal cancer. Okamoto and colleagues have reported that higher expression of IDO results in poorer prognosis and progression of serous type of ovarian cancer [31]. Very similar results were obtained in a later study by Takao et al. [32]. Finally, lower survival rate for patients with hepatocellular carcinoma was reported to have direct relation with higher level of IDO expression based on data reported by Pan and colleagues [33]. Thus, increasing evidence supports the hypothesis that enhancement of IDO activity may create a microenvironment in which effector immune response has been suppressed and tolerance mode towards tumor is increasing, thereby resulting in tumor growth and disease progression.

\section{IDO and transplantation}

The alleged IDO's capacity in induction of immune tolerance has provided a rationale to investigate the hypothesis that IDO plays an essential role in preventing graft rejection by creating an immune tolerance status. In fact, several studies have already demonstrated that enhancement of IDO activity in grafted tissue have protected the tissue against rejection. Recent findings suggested that upregulation of IDO in colon through an IFN $\gamma$ pathway resulted in T cells anergy (inactivity and lack of energy), suppression of immune responses and importantly, less injury in the colon and ameliorated lethality [34]. Studies by Ingelsten and her team suggest that IDO activity is partly contributing to long term acceptance of liver and kidney transplantation [35]. The precise mechanism(s) of this interaction has not been fully understood. However, two possible scenarios have been proposed. Based on the first scenario, induction of IDO metabolite, Kynurenine, is responsible for $\mathrm{T}$ cell anergy and suppressing Graft-VersusHost disease (GVHD), the main cause of mortality in certain transplantations. The second theory hypothesizes that induction of IDO by utilizing a TLR7/8 agonist minimized GVHD tissue injury and lethality. Another study offers the possibility that acute GVHD lethality may be blocked by myeloid cells in an IDO dependent fashion. All together, these observations are supporting the notion that proposed IDO tolerogenic feature has to be considered as a potential target to be included in a cell therapy protocol for treatment of GVHD.

IDO and autoimmune diseases

Increasing evidence supports the theory that IDO may delay the onset and progression of autoimmune diseases. A recent study showed that IDO-expressing natural killer (NK) cells contribute and promotes rat liver allograft acceptance [36]. Data from a study of a murine model of autoimmunity, experimental autoimmune encephalomyelitis (EAE), suggest that IDO may suppress $T$ cell activity associated with the different phases of this model and use of IDO inhibitor, 
$1 \mathrm{MT}$, resulted in increased inflammation, tissue damage and progression of disease [37]. Furthermore, recent study implies that IDO contributes to the immunomodulatory function of certain mesenchymal stem cells derived from human gingival tissue which seems to ameliorate inflammation-related tissue damage in experimental colitis [38]. Expression of IDO by synovial dendritic cells have been reported in patients with autoimmune rheumatoid arthritis (RA). From one viewpoint, IDO expression in RA patients is in agreement with the theory that IDO possesses immunosuppressive capacity and its presence is to control and contain the excessive $\mathrm{T}$ cell responses in RA patients. Therefore, inhibiting IDO activity will probably result in progression of disease [39, 40]. However, from another perspective, IDO paradoxically drives autoimmunity by stimulating B cells and surprisingly, using IDO inhibitor, 1MT, was able to reduce autoreactive B cell reaction [41]. The mechanism(s) of B cell activation through an IDO dependent pathway has not been fully understood; however, one assumption to explain this discrepancy in IDO function might be just because B cells are basically different cells from other APCs such as DCs, PDCs and macrophages. Therefore, it is possible that IDO interaction with B cells results in a different outcome compared with its interaction with non B cells. Other factors such as variation in genetic background of animal models, involvement of IDO2 or practical design of research protocol may be also responsible for varying observations.

IDO and diabetes: Can IDO be a predictive marker for diabetes?

Although, the role of IDO in autoimmune diseases including T1D has not been fully described yet, however, mounting evidence supports the assumption that IDO may contribute to a set of mechanisms and interactions which delays progression of autoimmune diseases and restores the static and tolerance barrier.

Recent studies have demonstrated the long term survival and viability of syngeneic islets exposed to IDO-expressing fibroblasts within the composite grafts in a diabetic animal model [42]. Data from other studies have provided encouraging evidence for treatment of T1D using IDO expressing encapsulated Sertoli cells [43]. Importantly, it has been shown that treatment with IDO inhibitor, 1MT, accelerated disease progression in T1D [44]. It has been also reported that a defect in tryptophan catabolism impairs tolerance in nonobese diabetic mice (NOD) [45]. Moreover, daily human chorionic gonadotropin injections significantly inhibited T1D onset in NOD female mice in an IDO dependent fashion [45]. Also, functional IDO was induced when human islets were treated with IFN $\gamma$. These findings suggest that IDO has the potential to be considered for assessment in any new therapeutic strategy for prognostic and therapeutic purposes.

Several reports have already examined the possibility of using IDO activity as a potential marker in predicting clinical outcomes as well as treatment efficiency. IDO activity is measured by the level of serum Kynurenin to Tryptophan $(\mathrm{K} / \mathrm{T})$ ratio. Kynurenin is the metabolite produced as a result of degradation of the essential amino acid L-tryptophan to $\mathrm{N}$-formylkynurenine. Several reports have noted a poor predicted survival rate in cancer patients with remarkable increase in the level of tryptophan degradation, higher level of kynurenine and elevated $\mathrm{K} / \mathrm{T}$ ratio. It is also observed that patients with acute kidney rejection have elevated $\mathrm{K} / \mathrm{T}$ ratio as early as one day after the graft. This suggests an anti-inflammatory role for IDO activity, although it might have been too late to overcome the $\mathrm{T}$ cell responses against the graft [46].

Taken together, these data clearly suggest that IDO and its activity in different tissues may be correlated with conditions and clinical outcomes of certain diseases. This is potentially a fascinating opportunity for a possible novel prognostic and therapeutic application; however, before reaching any comprehensive conclusion, numerous factors and variables in relation to each condition need to be investigated and clarified.

\section{Can IDO be used as a therapeutic target for diabetes?}

Different therapeutic strategies have been proposed in order to induce IDO expression in human DCs. Cellular therapies with human DCs, induced to express IDO ex vivo, offer a potential alternative approach that has the potential advantage of allowing defined antigens to be introduced into patients with heightened $\mathrm{T}$ cell responsiveness that drives autoimmune and allergic disease progression, and allograft rejection. However, technical difficulties in preparing clinical grade 'tolerogenic' DCs uniformly expressing IDO is one of the main practical problem with this protocol before being applied in practice or even at any clinical trials.

To have a successful IDO-dependent therapeutic protocol for autoimmune diseases, Penberthy suggests that pharmacological doses of NAD precursors (nicotinic acid/ niacin, nicotinamide/niacinamide, or nicotinamide riboside, with no tryptophan) have to be included in therapeutic regimens. This is because of evidence implying that autoimmune disease may partially be regarded as localized pellagra at the site of inflammation [47].

Another intriguing approach for IDO therapy is to develop genetic methods to express IDO ectopically in long-lived stromal (non-DC) cell types, or by introducing vectors into undifferentiated progenitor cells that continuously replenish DC populations, or other cell types that can suppress $\mathrm{T}$ cell mediated immunity when induced to 
express IDO. Some promising results have already emerged from this type of approach. Adenovirus and transposon based vectors engineered to express IDO ectopically protected donor lung allografts from $\mathrm{T}$ cell mediated rejection in rodent models, even in the complete absence of global immunosuppression $[48,49]$. Recently, Liu et al. introduced naked DNA encoding human IDO into rats prior to transferring their lungs into recipient mice, and showed that this genetic manipulation increased donor lung allograft survival significantly due to enhanced IDO activity in donor lungs [50]. Furthermore, IDO activity reduced, but did not completely prevent, the generation of allo-specific effector $\mathrm{T}$ cells that homed to donor lungs, and also impaired the cytotoxic functions of $\mathrm{T}$ cells in lung tissues [50].

IDO and immune system: a hypothetical mechanistic approach

The assumed immunoregulatory function of IDO on the one hand, and the autoimmune nature of T1D on the other hand, motivated us to hypothesize that IDO may be considered not only as a predictive marker, but also as a therapeutic target, which can be used in certain applications for restoring the immune tolerance and treatment of autoimmune diseases including T1D. To support our proposal, we suggest a mechanistic model to provide a preliminary platform for further investigations.

A new subset of effector T cells, Th17, are CD4+ T cells which are able to express pro-inflammatory cytokines including IL-17, IL-21 and IL-22. These cytokines are involved not only in host defense mechanisms against pathogens, but also, they are naturally contributing to pathogenesis of autoimmune diseases including T1D. The data from our recent study clearly suggested that IDO is a key regulator of Th17 cells. Inhibition of IDO by using IDO's pharmacologic inhibitor, 1MT, or utilizing IDO deficient mice was the key factor in conversion of stimulatory effector Th-17 like $\mathrm{T}$ cells to regulatory $\mathrm{T}$ cells (CD4+CD25+FOXP3+ T cells) [51]. These data along with pro-inflammatory capacity of IL-17 and its role in tissue damage, support the hypothesis that IDO may be used in certain therapeutic applications in treating T1D or other autoimmune diseases. Moreover, given the IL-17 potency in pathogenesis of autoimmunity, and the level of IL-17 expression may represent a biomarker to predict the clinical outcomes of autoimmune diabetes.

IDO can be induced by inflammatory stimuli such as Th1 cytokines or certain reagents such as soluble CTLA-4 (CTLA4-Ig) and TLR ligands. It has been shown that IDO can be expressed by a variety of cells including Antigen Presenting Cells (APC), such as dendritic cells (DCs), macrophages, and $\mathrm{B}$ cells as well as endothelial and epithelial cells [49-52]. A rare and specialized type of DCs in the peripheral blood and secondary lymphoid organs with an exclusive surface phenotype is called Plasmacytoid Dendritic Cells (PDCs), so named because of their plasma cell-like morphology [49, 53-55]. In human, PDCs are allegedly expressing CD123 and CCR6 while in mouse, they are characterized by expressing B220. Recent studies on murine model suggest that certain PDCs in bone marrow, blood, inflamed lymph nodes and secondary lymphoid organs express CD8, CD19 and IDO [56-58]. Several studies propose a dichotomy in PDCs function. It is assumed that they may be tolerogenic as well as immunologic [59]. That is why PDCs are allegedly referred to as key effectors in the innate immunity and a powerful potential target for immunotherapy of a variety of diseases including autoimmune diseases, cancer, ischemic reperfusion injury and transplantation [11, 49, 54, 60-63]. Recently, IDO expressing PDCs (Fig. 3) have become the center of many different studies focusing on treatment of autoimmune diseases including T1D. Saxena et al. [44] showed that depleting IDO expressing PDCs in NOD mice accelerated T1D progression while the onset of the disease and its severity were prevented by restoring IDO induction and PDCs function. Earlier studies by Grohmann and Fallarino also suggested that splenic DCs from prediabetic, T1D-prone female NOD mice have a defect in IDO
Fig. 3 Murine IDO expressing PDCs: A, In spleen, B, In culture, $(100 \mathrm{X})$
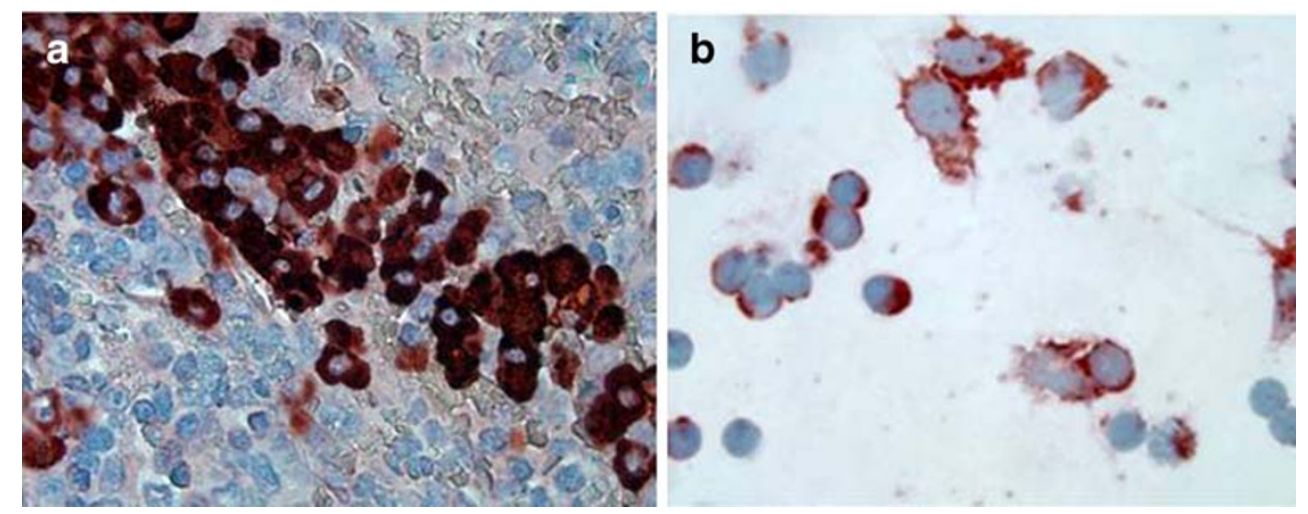
mediated $\mathrm{T}$ cell suppressor function which was reversed by a treatment which caused IDO induction [64, 65]. It is also important to mention that not all studies on PDCs have concluded a positive role for these cells in limiting the development of diseases and restoring the normal status. The study by $\mathrm{Li}$ et al. demonstrated an increase of IFN $\alpha$ expression in PDCs in the pancreatic lymph nodes showing the destructive impact of IFN $\alpha$ in the onset of T1D [66]. However, as Nikolic et al. have stated the diversity of IFN $\alpha$ producers may down play and minimize the role of PDCs in this necrotic reaction [52]. Importantly, data from another study by the same group, suggest that mice with elevated PDC/CDC ratio (CDC: Conventional Dendritic Cell) were significantly protected from diabetes. Therefore, this observation not only emphasizes the crucial role of PDCs in restoring immune tolerance and suppression of tissue damage, but also, it suggests that $\mathrm{PDC} / \mathrm{CDC}$ ratio may be used as an indicator factor to predict the clinical progression of diabetes as treatment goes on. As Nikolic et al. report [52], this finding is very similar to the results obtained by Ueda and colleagues, in which they showed an increased PDC/MDC ratio (MDC: Myeloid Dendritic Cells) was associated with intact immune tolerance during pregnancy [67].

Several studies have attempted to propose a mechanistic model by which PDCs may apply their assumed tolerogenic effects to result in induction of regulatory T cells [68-70].
One of these mechanisms is the alleged cross-talk between $\mathrm{T}$ regs and PDCs mediated by IDO. Based on this hypothetical model (Fig. 4), IDO expressing PDCs would be functioning in one of the 3 states of Reposing, Stimulating and Regulating thus they may reduce, in part, $\mathrm{T}$ cell mediated pathology in autoimmune diabetes.

In reposing state, immature PDCs take up and present selfantigens to $\mathrm{T}$ cells in a tolerogenic fashion, and regulatory cytokines such as TGF $\beta$ and IL-10 help maintain default counter-regulatory state.

Also, PDCs suppress standard DCs function and hold them in an immature status. In stimulating state, exposure to antigens (in case of T1D, self antigens) matures the PDCs, resulting in production of pro-inflammatory cytokines such as IL- 6 which initiate the conversion of resting regulatory $\mathrm{T}$ cells into effector Th17-like $\mathrm{T}$ cells expressing IL-17, a powerful inflammatory cytokine. In the regulating state, mature PDCs present antigen in a fashion which stimulate the generation of regulatory $\mathrm{T}$ cells with suppressor functions and in the meantime block the induction of pro-inflammatory cytokines and as a result ongoing onset of autoimmune diabetes will be abrogated and reversed. In regulatory state, IDO production by PDCs alters the stimulatory signals to anti-inflammatory pathways, suppressing inflammation and activate $\mathrm{T}$ regs to restore the tolerance which leads to control and ideally cure of the disease, in this case T1D.

\section{Regulating State Reposing State Stimulating State}

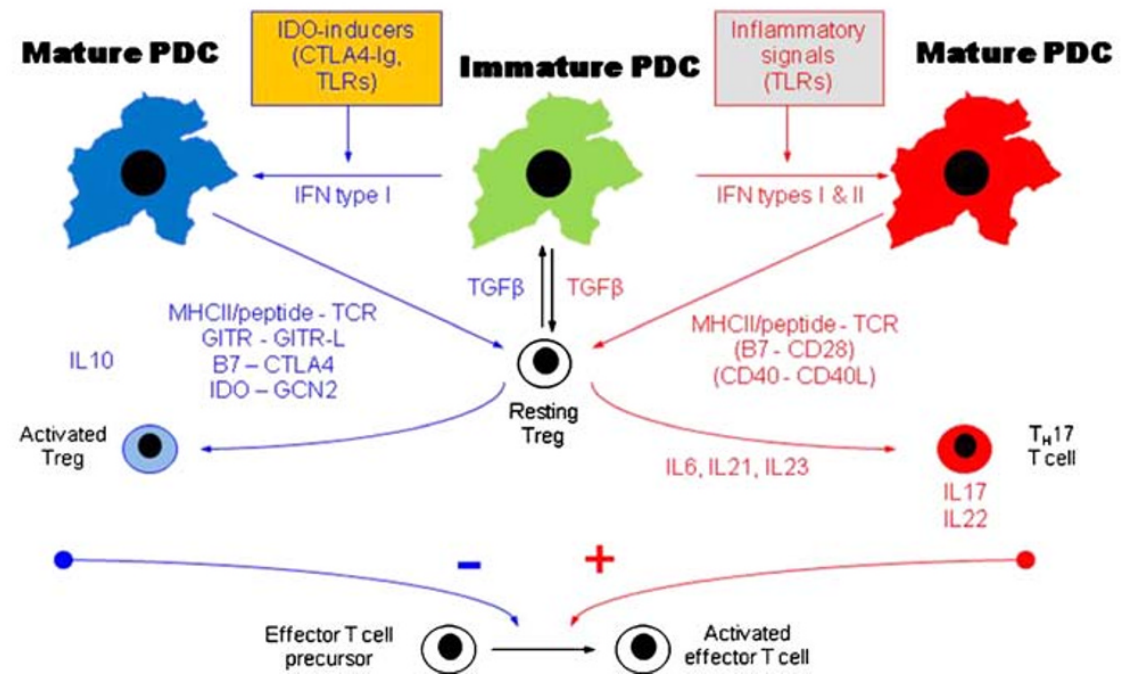

Fig. 4 A hypothetical mechanistic model for PDCs' function in the homeostasis, stimulatory and regulatory mode. Reposing State: In this state, immature PDCs introduce antigens in a tolerogenic manner and regulatory cytokines and factors such as TGF $\beta$ and IL10 help maintain tolerance. Stimulating State: In this state, mature PDCs and stimulatory $\mathrm{T}$ cells defeating tolerance barrier and pro-inflammatory cytokines such as IL6 promoting conversion of regulatory T cells into effector Th17-like Tcells expressing IL-17 and T1D develops. Regulating State : Mature PDCs provoke abortive T cell responses, activate $\mathrm{T}$ regs and suppress inflammatory cytokine production, mediated by IDO 


\section{Concluding remarks and future directions}

The theoretic ability of IDO to induce immune tolerance presents an extensive and promising horizon in treatment of autoimmune diseases such as T1D and conditions where tolerance barrier is overcome. It is clear that IDO production is not the only pathway by which PDCs exert their tolerogenic function in diabetes. However, the assumptive tolerogenic capacity of IDO indicates that IDO may serve as a potential and reliable prognostic indicator for early detection of diseases including autoimmune diabetes. The proposed crucial role of IDO in conversion of regulatory $\mathrm{T}$ cells into stimulatory Th-17 like cells and production of pro-inflammatory cytokines such as IL-6 and IL-17 offer added advantage as predictive for early detection of diseases such as T1D.

It is important to emphasize the fact that in some studies, IDO has generated very paradoxical results. In other words, any application of IDO may be associated with limitations. In fact, the dichotomy in IDO's function is a major potential limiting factor. This means that IDO's impact on a biological system could be variable based on several factors such as conditions of the microenvironment, target cells, IDO's capacity and pathologic status of the disease. Another important limiting factor for IDO application is IDO-2. This novel gene with homology to IDO has the capacity to encode an enzyme to catabolism tryptophan. Despite similarities, IDO-2 may act differently from IDO-1 in reaction to both stimuli and inhibitors.

Taken together, data presented in this article offers a rationalized platform to investigate the hypothesis that IDO may have the capacity of acting as a prognostic and therapeutic factor in prediction and treatment of autoimmune diseases, particularly T1D. Current and future studies on IDO should consider both proposed capabilities and limitations of IDO. Given the encouraging preliminary findings in restoring immune tolerance and inhibition of pathologic progression of autoimmune disease, IDO has the potential to represent a fundamental mechanism in maintaining tolerance and suppression of tissue damage as a result of immune hyperactivity.

Acknowledgments The authors thank Drs. David Munn, Andrew Mellor and Mr. Burles Johnson (Immunotherapy Center, Medical College of Georgia) for critical comments.

Conflict of interest The authors declare that they have no conflict or competing interests.

\section{References}

1. Sugimoto H, Oda S, Otsuki T, et al. Crystal structure of human indoleamine 2, 3-dioxygenase: catalytic mechanism of $\mathrm{O} 2$ incorporation by a heme-containing dioxygenase. Proc Natl Acad Sci USA. 2006;103(8):2611-6.

2. Ball HJ, Sanchez-Perez A, Weiser S, et al. Characterization of an indoleamine 2,3-dioxygenase-like protein found in humans and mice. Gene 2007;396(1):203-13.

3. Ball HJ, Yuasa HJ, Austin CJ, et al. Indoleamine 2, 3dioxygenase-2; a new enzyme in the kynurenine pathway. Int $\mathrm{J}$ Biochem Cell Biol. 2009;41(3):467-71.

4. Metz R, Duhadaway JB, Kamasani U, et al. Novel tryptophan catabolic enzyme IDO2 is the preferred biochemical target of the antitumor indoleamine 2, 3-dioxygenase inhibitory compound D1-methyl-tryptophan. Cancer Res. 2007;67(15):7082-7.

5. Hayashi T, Beck L, Rossetto C, et al. Inhibition of experimental asthma by indoleamine 2, 3-dioxygenase. J Clin Invest. 2004;114 (2):270-9.

6. Baban B, Chandler P, McCool D, et al. Indoleamine 2, 3dioxygenase expression is restricted to fetal trophoblast giant cells during murine gestation and is maternal genome specific. J Reprod Immunol. 2004;61(2):67-77.

7. Beutelspacher SC, Tan PH, McClure MO, et al. Expression of indoleamine 2, 3-dioxygenase (IDO) by endothelial cells: implications for the control of alloresponses. Am J Transplant. 2006;6 (6): $1320-30$

8. Mohib K, Wang S, Guan Q, et al. Indoleamine 2, 3-dioxygenase expression promotes renal ischemia-reperfusion injury. Am J Physiol Renal Physiol. 2008;295(1):F226-34.

9. Chen Y, Guillemin GJ. Kynurenine Pathway Metabolites in Humans: Disease and Healthy States. Int $\mathrm{J}$ Tryptophan Res. 2009;2:1-19.

10. Littlejohn TK, Takikawa O, Truscott RJ, et al. Asp274 and his346 are essential for heme binding and catalytic function of human indoleamine 2, 3-dioxygenase. J Biol Chem. 2003;278(32):29525-31.

11. Johnson BA, Baban B, Mellor AL (2009) Targeting the immunoregulatory Indoleamine 2,3-dioxygenase pathway in Immunotherapy. Immunotherapy 1(4)

12. Pfefferkorn ER. Interferon gamma blocks the growth of Toxoplasma gondii in human fibroblasts by inducing the host cells to degrade tryptophan. Proc Natl Acad Sci USA. 1984;81(3):908-12.

13. Munn DH, Zhou M, Attwood JT, et al. Prevention of allogeneic fetal rejection by tryptophan catabolism. Science 1998;281 (5380):1191-3.

14. Müller A, Heseler K, Schmidt SK, et al. The missing link between indoleamine 2,3-dioxygenase mediated antibacterial and immunoregulatory effects. J Cell Mol Med. 2009;13(6):1125-35.

15. Spekker K, Czesla M, Ince V, et al. Indoleamine 2, 3-dioxygenase is involved in defense against Neospora caninum in human and bovine cells. Infect Immun. 2009;77(10):4496-501.

16. Curti A, Trabanelli S, Salvestrini V, et al. The role of indoleamine 2, 3-dioxygenase in the induction of immune tolerance: focus on hematology. Blood 2009;113(11):2394-401.

17. Kaufmann SH. Immunity to intracellular bacteria. Annu Rev Immunol. 1993;11:129-63.

18. Yoshida R, Hayaishi O. Induction of pulmonary indoleamine 2, 3dioxygenase by intraperitoneal injection of bacterial lipopolysaccharide. Proc Natl Acad Sci USA. 1978;75:3998-4000.

19. Däubener W, Spors B, Hucke C, et al. Restriction of Toxoplasma gondii growth in human brain microvascular endothelial cells by activation of indoleamine 2, 3-dioxygenase. Infect Immun. 2001;69:6527-31.

20. Mackler AM, Barber EM, Takikawa O, et al. Indoleamine 2, 3dioxygenase is regulated by IFN-gamma in the mouse placenta during Listeria monocytogenes infection. J Immunol. 2003;170: 823-30.

21. Yoshida R, Urade Y, Tokuda M, et al. Induction of indoleamine 2, 3-dioxygenase in mouse lung during virus infection. Proc Natl Acad Sci USA. 1979;76:4084-6. 
22. Bonifazi P, Zelante T, D'Angelo C, De Luca A, Moretti S, Bozza $\mathrm{S}$, et al. Balancing inflammation and tolerance in vivo through dendritic cells by the commensal Candida albicans. Mucosal Immunol. 2009;2(4):362-74. Epub 2009 May 6.

23. Zelante T, Fallarino F, Bistoni F, Puccetti P, Romani L. Indoleamine 2,3-dioxygenase in infection: the paradox of an evasive strategy that benefits the host. Microbes Infect. 2009;11(1):13341. Epub 2008 Oct 25.

24. Uyttenhove C, Pilotte L, Théate I, et al. Evidence for a tumoral immune resistance mechanism based on tryptophan degradation by indoleamine 2, 3-dioxygenase. Nat Med. 2003;9(10):1269-74.

25. Munn DH, Sharma MD, Hou D, et al. Expression of indoleamine 2,3-dioxygenase by plasmacytoid dendritic cells in tumor-draining lymph nodes. J Clin Invest. 2004;114(2):280-90. Erratum in: J Clin Invest 114(4):599.

26. Muller AJ, DuHadaway JB, Donover PS, et al. Inhibition of indoleamine 2, 3-dioxygenase, an immunoregulatory target of the cancer suppression gene Bin1, potentiates cancer chemotherapy. Nat Med. 2005;11:312-9.

27. Gao YF, Peng RQ, Li J, et al. The paradoxical patterns of expression of indoleamine 2, 3-dioxygenase in colon cancer. $\mathrm{J}$ Transl Med. 2009;7:71.

28. Lee JR, Dalton RR, Messina JL, et al. Pattern of recruitment of immunoregulatory antigen-presenting cells in malignant melanoma. Lab Invest. 2003;83(10):1457-66.

29. Urakawa H, Nishida Y, Nakashima H et al (2009) Prognostic value of indoleamine 2,3-dioxygenase expression in high grade osteosarcoma. Clin Exp Metastasis [Epub ahead of print]

30. Brandacher G, Perathoner A, Ladurner R, et al. Prognostic value of indoleamine 2, 3-dioxygenase expression in colorectal cancer: effect on tumor-infiltrating T cells. Clin Cancer Res. 2006;12 (4):1144-51.

31. Okamoto A, Nikaido $\mathrm{T}$, Ochiai $\mathrm{K}$, et al. Indoleamine 2, 3dioxygenase serves as a marker of poor prognosis in gene expression profiles of serous ovarian cancer cells. Clin Cancer Res. 2005;11(16):6030-9.

32. Takao M, Okamoto A, Nikaido T, et al. Increased synthesis of indoleamine-2,3-dioxygenase protein is positively associated with impaired survival in patients with serous-type, but not with other types of, ovarian cancer. Oncol Rep. 2007;17(6):1333-9.

33. Pan K, Wang H, Chen MS, et al. Expression and prognosis role of indoleamine 2, 3-dioxygenase in hepatocellular carcinoma. J Cancer Res Clin Oncol. 2008;134(11):1247-53.

34. Jasperson LK, Bucher C, Panoskaltsis-Mortari A, et al. Inducing the tryptophan catabolic pathway, indoleamine 2, 3-dioxygenase (IDO), for suppression of graft-versus-host disease (GVHD) lethality. Blood 2009;114(24):5062-70.

35. Ingelsten M, Gustafsson K, Oltean M, et al. Is indoleamine 2, 3dioxygenase important for graft acceptance in highly sensitized patients after combined auxiliary liver-kidney transplantation? Transplantation 2009;88(7):911-9.

36. Wang C, Tay SS, Tran GT et al Donor IL-4-treatment induces alternatively activated liver macrophages and IDO-expressing NK cells and promotes rat liver allograft acceptance. Transpl Immunol [Epub ahead of print]

37. Platten M, Ho PP, Youssef S, et al. Treatment of autoimmune neuroinflammation with a synthetic tryptophan metabolite. Science 2005;310(5749):850-5. Erratum in: Science. 2006 Feb 17;311(5763):954.

38. Zhang Q, Shi S, Liu Y, et al. Mesenchymal stem cells derived from human gingiva are capable of immunomodulatory functions and ameliorate inflammation-related tissue destruction in experimental colitis. J Immunol. 2009;183(12):7787-98.

39. Zhu L, Ji F, Wang Y, et al. Synovial autoreactive $T$ cells in rheumatoid arthritis resist IDO-mediated inhibition. J Immunol. 2006;177:8226-33.
40. Takakubo Y, Takagi M, Maeda K, et al. Distribution of myeloid dendritic cells and plasmacytoid dendritic cells in the synovial tissues of rheumatoid arthritis. J Rheumatol. 2008;35(10):1919 31.

41. Scott GN, DuHadaway J, Pigott E, et al. The immunoregulatory enzyme IDO paradoxically drives B cell-mediated autoimmunity. J Immunol. 2009;182(12):7509-17.

42. Jalili RB, Forouzandeh F, Moeenrezakhanlou A, et al. Mouse pancreatic islets are resistant to indoleamine 2, 3 dioxygenaseinduced general control nonderepressible-2 kinase stress pathway and maintain normal viability and function. Am J Pathol. 2009; 174(1):196-205.

43. Fallarino F, Volpi C, Zelante T, et al. IDO mediates TLR9-driven protection from experimental autoimmune diabetes. J Immunol. 2009;183(10):6303-12.

44. Saxena V, Ondr JK, Magnusen AF, et al. The countervailing actions of myeloid and plasmacytoid dendritic cells control autoimmune diabetes in the nonobese diabetic mouse. J Immunol. 2007;179(8):5041-53.

45. Ueno A, Cho S, Cheng L, et al. Transient upregulation of indoleamine 2, 3-dioxygenase in dendritic cells by human chorionic gonadotropin downregulates autoimmune diabetes. Diabetes 2007;56(6):1686-93.

46. Brandacher G, Cakar F, Winkler C, et al. Non-invasive monitoring of kidney allograft rejection through IDO metabolism evaluation. Kidney Int. 2007;71(1):60-7.

47. Penberthy WT. Pharmacological targeting of IDO-mediated tolerance for treating autoimmune disease. Curr Drug Metab. 2007;8(3):245-66.

48. Swanson KA, Zheng Y, Heidler KM, et al. CDllc+ cells modulate pulmonary immune responses by production of indoleamine 2, 3-dioxygenase. Am J Respir Cell Mol Biol. 2004; 30:311-8

49. Liu H, Liu L, Liu K, et al. Novel action of indoleamine 2, 3 dioxygenase attenuating acute lung allograft injury. Am J Respir Crit Care Med. 2006;173:566-72.

50. Liu H, Liu L, Liu K, et al. Reduced cytotoxic function of effector $\mathrm{CD} 8+\mathrm{T}$ cells is responsible for indoleamine 2, 3-dioxygenasedependent immune suppression. J Immunol. 2009;183:1022-31.

51. Baban B, Chandler PR, Sharma MD, et al. IDO activates regulatory $\mathrm{T}$ cells and blocks their conversion into Th17-like $\mathrm{T}$ cells. J Immunol. 2009;183(4):2475-83.

52. Nikolic T, Welzen-Coppens JM, Leenen PJ, et al. Plasmacytoid dendritic cells in autoimmune diabetes-potential tools for immunotherapy. Immunobiology 2009;214(9-10):791-9.

53. Steinman RM, Banchereau J. Taking dendritic cells into medicine. Nature 2007;449(7161):419-26.

54. Kadowaki N. The divergence and interplay between pDC and $\mathrm{mDC}$ in humans. Front Biosci. 2009;14:808-17.

55. Kadowaki N. Dendritic cells: a conductor of T cell differentiation. Allergol Int. 2007;56(3):193-9.

56. Mellor AL, Munn DH. Creating immune privilege: active local suppression that benefits friends, but protects foes. Nat Rev Immunol. 2008;8(1):74-80. Erratum in: Nat Rev Immunol. 2008 Feb;8(2): 160 .

57. Nestle FO, Conrad C, Tun-Kyi A, et al. Plasmacytoid predendritic cells initiate psoriasis through interferon-alpha production. J Exp Med. 2005;202(1):135-43.

58. Pechhold K, Koczwara K. Immunomodulation of autoimmune diabetes by dendritic cells. Curr Diab Rep. 2008;8(2):107-13.

59. Munn DH, Sharma MD, Lee JR, et al. Potential regulatory function of human dendritic cells expressing indoleamine 2, 3dioxygenase. Science 2002;297(5588):1867-70.

60. Fallarino F, Vacca C, Orabona C, et al. Functional expression of indoleamine 2, 3-dioxygenase by murine CD8 alpha $(+)$ dendritic cells. Int Immunol. 2002;14(1):65-8. 
61. Baban B, Hansen AM, Chandler PR, et al. A minor population of splenic dendritic cells expressing CD19 mediates IDO-dependent T cell suppression via type I IFN signaling following B7 ligation. Int Immunol. 2005;17(7):909-19.

62. Jaehn PS, Zaenker KS, Schmitz J, et al. Functional dichotomy of plasmacytoid dendritic cells: antigen-specific activation of $\mathrm{T}$ cells versus production of type I interferon. Eur J Immunol. 2008;38 (7): $1822-32$.

63. Steinman RM, Banchereau J. Taking dendritic cells into medicine. Nature 2007;449(7161):419-26.

64. Grohmann U, Fallarino F, Bianchi R, et al. Tryptophan catabolism in nonobese diabetic mice. Adv Exp Med Biol. 2003; 527:47-54.

65. Fallarino F, Luca G, Calvitti M, et al. Therapy of experimental type 1 diabetes by isolated Sertoli cell xenografts alone. J Exp Med. 2009;206(11):2511-26.
66. Li Q, Xu B, Michie SA, et al. Interferon-alpha initiates type 1 diabetes in nonobese diabetic mice. Proc Natl Acad Sci USA. 2008;105(34):12439-44.

67. Ueda Y, Hagihara M, Okamoto A, et al. Frequencies of dendritic cells (myeloid DC and plasmacytoid DC) and their ratio reduced in pregnant women: comparison with umbilical cord blood and normal healthy adults. Hum Immunol. 2003;64(12):1144-51.

68. Sharma MD, Baban B, Chandler P, et al. Plasmacytoid dendritic cells from mouse tumor-draining lymph nodes directly activate mature Tregs via indoleamine 2, 3-dioxygenase. J Clin Invest. 2007;117(9):2570-82.

69. Colonna M, Trinchieri G, Liu YJ. Plasmacytoid dendritic cells in immunity. Nat Immunol. 2004;5(12):1219-26.

70. Soumelis V, Liu YJ. From plasmacytoid to dendritic cell: morphological and functional switches during plasmacytoid pre-dendritic cell differentiation. Eur J Immunol. 2006;36(9):2286-92. 